\title{
Performance Evaluation of a Cooperative OFDM System with implementation of DAF and AAF Relaying Protocols on Color image transmission
}

\author{
M Mowrin Hossain and Shaikh Enayet Ullah \\ Department of Information and Communication Engineering, University of Rajshahi \\ Rajshahi-6205, Bangladesh \\ mowrin_3@yahoo.com , enayet67@yahoo.com
}

\begin{abstract}
In this paper, we investigate the performance of a single-relay cooperative wireless communication system with implementation of Orthogonal Frequency division multiplexing technique under Amplifyand-Forward $(A A F)$ and Decode-and-Forward $(D A F)$ relaying protocols on color image transmission. The system under investigation incorporates four conventional signal combining schemes such as Equal Ratio Combining (ERC) and Fixed Ratio Combining (FRC), Signal to Noise Ratio Combining (SNRC) and Enhanced Signal to Noise Ratio Combining (ESNRC) under BPSK and QPSK digital modulations. In the present study, results of BER simulation in AWGN and Raleigh fading channels shows that the system outperforms in ERC combining scheme as compared to others (FRC, SNRC and ESNRC) under BPSK digital modulation and AAF relaying protocol. It is observed that a quite satisfactory system performance is achieved in ERC scheme under AAF relaying protocol with BPSK digital modulation. It has been anticipated from the simulation study that the performance of the cooperative OFDM communication system degrades with the increasing of noise power and level of modulation.
\end{abstract}

\section{Keywords}

Relaying protocol, Cooperative OFDM system, Signal Combining scheme, Bit Error rate, AWGN and Raleigh fading channels.

\section{Introduction}

Cooperative and relay communications have been one of the most widely explored topics in communications over the past few years. The key idea is to have users cooperate in transmitting their messages to the destination, instead of operating independently and competing among each other for channel resources, as done in conventional networks. However, as the field has progressed over the years, cooperative communications have now become a design concept rather than a specific transmission technology. This concept has revolutionized the design of wireless networks, allowing us to increase coverage, throughput, and transmission reliability even as conventional transmission techniques gradually reach their limits. In recent years, cooperative and relay technologies are expected to be implemented in next generation wireless communication system targeted to achieve 1Gbps for downlink (DL) and $500 \mathrm{Mbps}$ for uplink (UL) throughput under LTE-Advanced standard (Peter et al., 2010; Young et al., 2010). In wireless Communication systems, the use of diversity techniques has by now been well documented to combat the detrimental effects of signal fading. Cooperative DOI : $10.5121 /$ ijitcs.2011.1303 
communications are gaining increasing interest in the wireless community as a new diversity enabler. In cooperative communications, besides the source destination pair, some cooperating nodes that overhear the source node are also involved in the signal transmission by relaying the received signal from the source to the destination. Cooperative communications can achieve spatial diversity because signals bearing the same information go through uncorrelated channels introduced by cooperating nodes. Although spatial diversity can also be achieved by multiple input multiple output (MIMO) technology, nodes in cooperative networks need not be equipped with multiple antennas and thus require less hardware complexity while offering counter-fading capability. Moreover, cooperative communication can also mitigate the effects of shadowing and path loss inherent in wireless channels. It has been shown that cooperative communications not only increase the data rate but also reduce error probability of wireless links (Sendonaris et al., 2003(a); Sendonaris et al., 2003(b); Laneman et al., 2004).

\section{Materials and Methods}

We assume that the simulated Cooperative OFDM system implements two relaying protocols (AAF and DAF) .A description of these protocols are given below.

\subsection{Relaying Protocols}

\subsubsection{Amplify-and-Forward (AAF)}

In amplify-and-forward (AAF) relaying schemes, the relay forwards a scaled version of the received signal to the destination regardless of the source-relay link quality. In Phase I, the source transmits the symbol block

$\mathrm{X}_{s}=\left[x_{s}[0], \ldots, x_{s}[M-1]\right]$ to both the relay and the destination and the received signals are given by

$y_{r}[m]=h_{s, r} \sqrt{P_{s}} x_{s}[m]+w_{r}[m]$,

$y_{d}^{(1)}[m]=h_{s, d} \sqrt{P_{s}} x_{s}[m]+w_{d}^{(1)}[m]$,

respectively for $m=0,1 \ldots M-1$, Here, $P_{s}$ is the source transmission power, $h_{s, r}$ and $h_{s, d}$ are the channel coefficients of the source-to-relay (s-r) and the source-to-destination (s-d) links,respectively, and $\mathrm{w}_{\mathrm{r}}[\mathrm{m}] \sim C N\left(0, \sigma_{\mathrm{r}}^{2}\right)$ and $\mathrm{w}_{\mathrm{d}}{ }^{(1)}[\mathrm{m}] \sim C N\left(0, \sigma_{\mathrm{d}}^{2}\right)$ are the additive white Gaussian noise (AWGN) at the relay and the destination, respectively.

In Phase II, the relay first scales the received signal in (1) to yields a normalized transmit vector $x_{r}$ with $\left.\left.\mathrm{E}|| x_{r}[m]\right|^{2}\right]=1$, for all $\mathrm{m}$. If the instantaneous channel gain $\left[h_{s, r}\right]^{2}$ is known at the relay, the relay can multiply the received signal $y_{r}[m]$ by the gain

$$
G_{v}=\frac{1}{\sqrt{\mathrm{E}\left[\left.\left|\mathrm{y}_{\mathrm{r}}[m]\right|^{2}|| \mathrm{h}_{\mathrm{s}, \mathrm{r}}\right|^{2}\right]}}=\frac{1}{\sqrt{\mathrm{P}_{\mathrm{s}}\left|\mathrm{h}_{\mathrm{s}, \mathrm{r}}\right|^{2}+\sigma_{\mathrm{r}}^{2}}}
$$

and we can write,

$x_{r}[m]=G_{v} y_{r}[m]$ 
International Journal of Information Technology Convergence and Services (IJITCS) Vol.1, No.3, June 2011

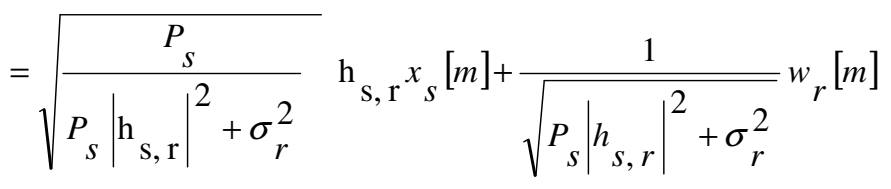

It is noticeable that the gain $G_{v}$ depends on the source relay channel coefficient $h_{s, r}$

The relay forwards the signal $x_{r}$ with power $P_{r}$ to the destination where the received signal can be expressed as

$$
\begin{aligned}
y_{d}^{(2)}[m] & =h_{r, d} \sqrt{P_{r}} x_{r}[m]+w_{d}^{(2)}[m] \\
& =\sqrt{\frac{P_{s} P_{r}}{\left.P_{s \mid} h_{s, r}\right|^{2}+\sigma_{r}^{2}}} \mathrm{~h} \mathrm{~s}, \mathrm{r}_{r, d}{ }_{r,{ }_{s}}[m]+\sqrt{\frac{P_{r}}{P_{s}\left|h_{s, r}\right|^{2}+\sigma_{r}^{2}}} \mathrm{~h}_{\mathrm{r}, \mathrm{d}} w_{r}[m]+w_{d}^{(2)}[m]
\end{aligned}
$$

For $\mathrm{m}=\mathrm{m}=0 . . \mathrm{M}-1$. The signals arriving at the destination can be utilized for detection with or without diversity combining.

\subsubsection{Decode-and-Forward (DAF)}

In Decode-and-forward (DAF) relaying schemes, the source first transmits a message to both the relay and the destination in Phase I. Then, if the relay is able to successfully decode the message, it will regenerate the same message and forward it to the destination in Phase II. It is assumed that in each cooperative transmission, an equal amount of time is allocated to Phase I and Phase II. Specifically, in Phase I, the source transmits the symbol block to both the relay and the destination. Due to the broadcast nature of the wireless medium, both the relay and the destination will receive a noisy version of the signal in Phase I, which are identical to equation 1 and 2.In phase II, the relay correctly decodes the message and re-encodes it into a codeword and retransmits it to the destination (Peter et al.,2010).

In receiving section of the wireless communication system, the transmitted signal following independent fading channels for multiple receive antennas are co phased and combined to obtain a resultant signal using various signal combining schemes as follows (Leng et al., 2010):

\subsection{Combining Techniques}

\subsubsection{Equal ratio combining (ERC)}

In ERC, the weighting coefficients $\mathrm{k}_{1}=\mathrm{k}_{2}=\mathrm{k}_{3}=\cdots \mathrm{k}_{\mathrm{N}}$, the signal received at destination is given by:

$$
y_{d}[n]=\sum_{\mathrm{i}=1}^{\mathrm{k}} \mathrm{y}_{\mathrm{i}, \mathrm{d}}[n]
$$

and in case of single relay, the equation (6) is modified to:

$$
y_{d}[n]=\mathrm{y}_{\mathrm{s}, \mathrm{d}}[n]+y_{r, d}[n]
$$

Where, $y_{s, d}[n]$ is the signal from the source node to the destination node. $y_{r, d}[n]$ is the signal from the relay to the destination node. 


\subsubsection{Fixed ratio combining (FRC)}

In FRC, the received signals are combined based on a fixed weighting factor instead of being added together averagely, while the weighting factor in the whole process of the communication is not changing. The weighting coefficients need to be determined by the channel fading and other factors. The signal received at destination in FRC can be expressed as

$$
y_{d}[n]=\sum \mathrm{d}_{\mathrm{i}, \mathrm{d}} \cdot y_{i, d}[n]
$$

Here, $d_{i, d}$ is the received signal, $y_{i, d}$ is the weighting factor, if only one relay, then the equation (8) can be written as:

$$
y_{d}[n]=\mathrm{d}_{\mathrm{s}, \mathrm{d}} \cdot y_{s, d}[n]+d_{s, r, d} \cdot y_{r, d}[n]
$$

Where, $d_{s, d}$ is the weighting coefficient of a direct link and $d_{s, r, d}$ is the weighting coefficient of the relay link.

\subsubsection{Signal to noise ratio combining (SNRC)}

In SNRC, the weighting factor is based on the SNR of the received signal. The combined signal can be obtained as

$$
y_{d}[n]=\sum_{i=1}^{\mathrm{k}} \mathrm{SNR}_{\mathrm{i}} \cdot y_{i, d}[n]
$$

and in case of single relay, the equation (10) is modified to:

$$
y_{d}[n]=\operatorname{SNR}_{\mathrm{s}, \mathrm{d}} \cdot y_{s, d}[n]+S N R_{, r, d} \cdot y_{r, d}[n]
$$

Where, $S N R_{s, d}$ is the weighting coefficient of a direct link and $S N R_{s, r, d}$ is the weighting coefficient of a relay link.

\subsubsection{Enhanced Signal to Noise Combining (ESNRC)}

Another plausible signal combining scheme is to ignore an incoming signal when the data from the other incoming channels have a much better quality. If the channels have more or less the same channel quality, the incoming signals are rationed equally. We can fix the SNR value of the channel for which this decision can be made. In this simulation study, a SNR value of $10 \mathrm{~dB}$ has been fixed up. The signal received at the destination can be expressed as

$$
y_{d}[n]=\left\{\begin{array}{cl}
y_{s, d}[n] & \frac{S N R_{s, d}}{S N R_{s, r, d}}>10 \\
y_{s, d}[n]+y_{s, r, d}[n] & 0.1 \leq \frac{S N R_{s, d}}{S N R_{s, r, d}} \leq 10 \\
y_{s, r, d}[n] & \frac{S N R_{s, d}}{S N R_{s, r, d}}<0.1
\end{array}\right.
$$

Where, $\mathrm{SNR}_{\mathrm{s}, \mathrm{d}}$ and $\mathrm{SNR}_{\mathrm{s}, \mathrm{r}, \mathrm{d}}$ are the sender to destination channel (direct channel) and the sender-relay-destination channel (relay channel) SNR values respectively( Laneman et al., 2004). 


\section{System Model}

The block diagram of the simulated Cooperative OFDM system is shown in Figure 1. It consists of three major units such as sender, relay and destination. In sender unit, a noncompressed digital color (RGB) image in Joint Photographic Experts Group (JPEG) format is used. as input information source. and it is discredited into raster of $160 \times 108$ picture elements and the analog transmission signals RGB are converted into digital image components after analog-to-digital conversion. The digital image samples of each RGB components are multiplexed serially (Tomas, 2003). The multiplexed binary bit stream is applied to the $1 / 2$-rated Convolutional encoder and interleaved (Goldsmith, 2005). The convolutionally encoded interleaved bits are modulated digitally by binary phase shift keying (BPSK)/ Quadrature phase shift keying (QPSK). The symbols are then fed into OFDM modulator which performs an IFFT on each OFDM block of length 256 symbols/ sub carriers followed by a parallel to serial conversion A cyclic prefix (CP) of length $L c p$ containing a copy of the last $L c p$ samples of the parallel to serial converted output of the 256-point IFFT is then prepended. The CP is essentially a guard interval, which serves to eliminate interference between OFDM symbols. However, the resulting OFDM symbols of length $256+L c p$ are lunched from the transmit antenna. At destination, the receiver receives two signals, one directly transmitted from sender and another one via relay. In relay unit, it receives transmitted signal from sender, it process the signal under any one of the two relaying protocols and re-transmits. In destination, the two signals are combined using various combining techniques and the transmitted signal from sender with its increased strength as compared to noise signal is retrieved. The retrieved signal processed through differ-rent sections of OFDM demodulator, is de-mapped, deinterleaved and convolutionally decoded to recover the transmitted input data.

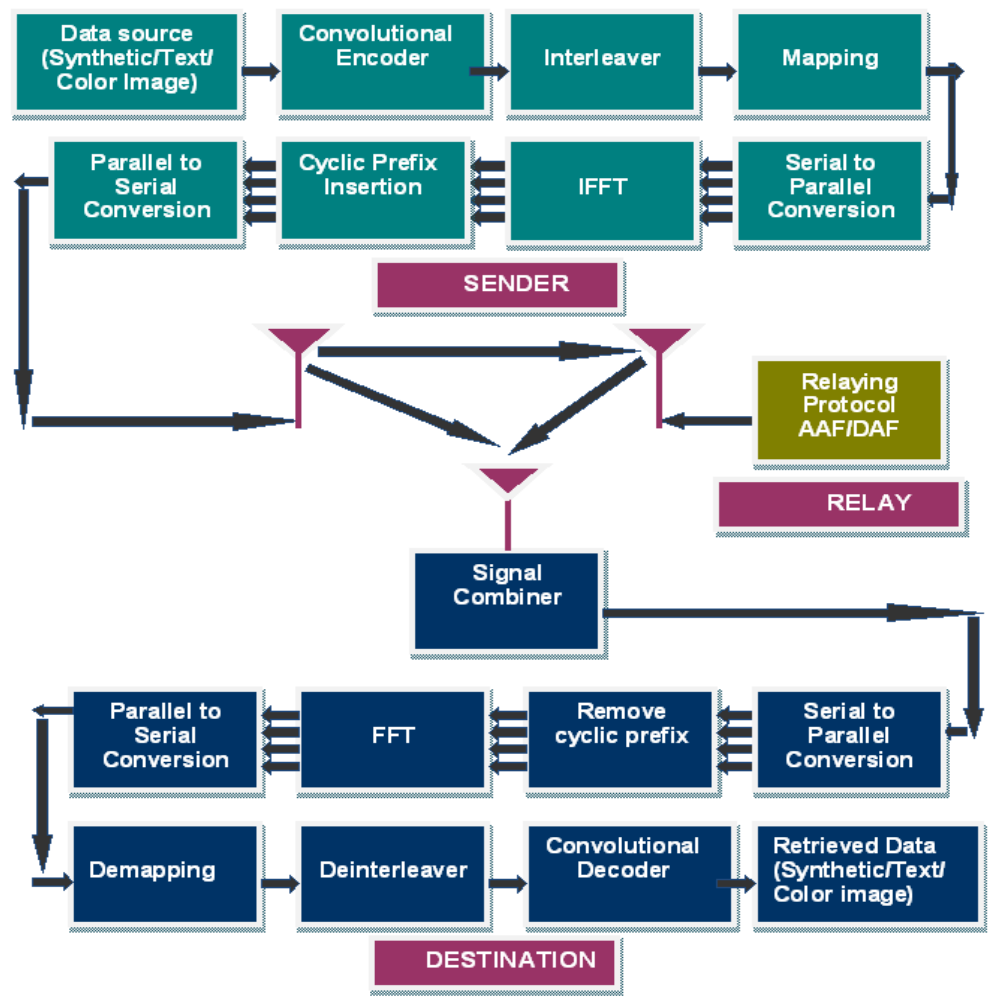

Figure 1: Block diagram of a Cooperative OFDM wireless Communication System 


\section{Results and Discussion}

The plotted curves presented in Figure 2 show a remarkable performance enhancement under Equal Ratio Combining scheme with AAF relaying protocol and BPSK digital modulation.For a typically assumed SNR value of $4 \mathrm{~dB}$, the BER values are 0.00001 and 0.00010 in case of ERC and FRC combining schemes viz, the system performance is improved in ERC by 20.00 $\mathrm{dB}$ as compared to FRC. In Figure 3, the system provides a quite satisfactory performance under Equal Ratio Combining scheme with DAF relaying protocol and BPSK digital modulation. At SNR value of $4 \mathrm{~dB}$, the BER is found to have a value of 0.0001 which is indicative of better system performance in BPSK modulation. In Figure 3, the system provides a quite satisfactory performance under Equal Ratio Combining scheme with DAF relaying protocol and BPSK digital modulation. At SNR value of $4 \mathrm{~dB}$, the BER is found to have a value of 0.0001 , which is indicative of better system performance in BPSK modulation. In Figure 4, the system provides a quite satisfactory performance under Equal Ratio Combining scheme with DAF relaying protocol and BPSK digital modulation. At low SNR value of $2 \mathrm{~dB}$, the BER value is 0.00003 which is high as compared to 0.00001 in case of AAF relaying protocol (Figure 2). In Figure 4, it is quite noticeable that an almost flat responsive system performance is observed in FRC relaying protocol. In case of SNRC and ESNRC signal combining schemes, the system shows almost identical response over a significant band of SNR values.

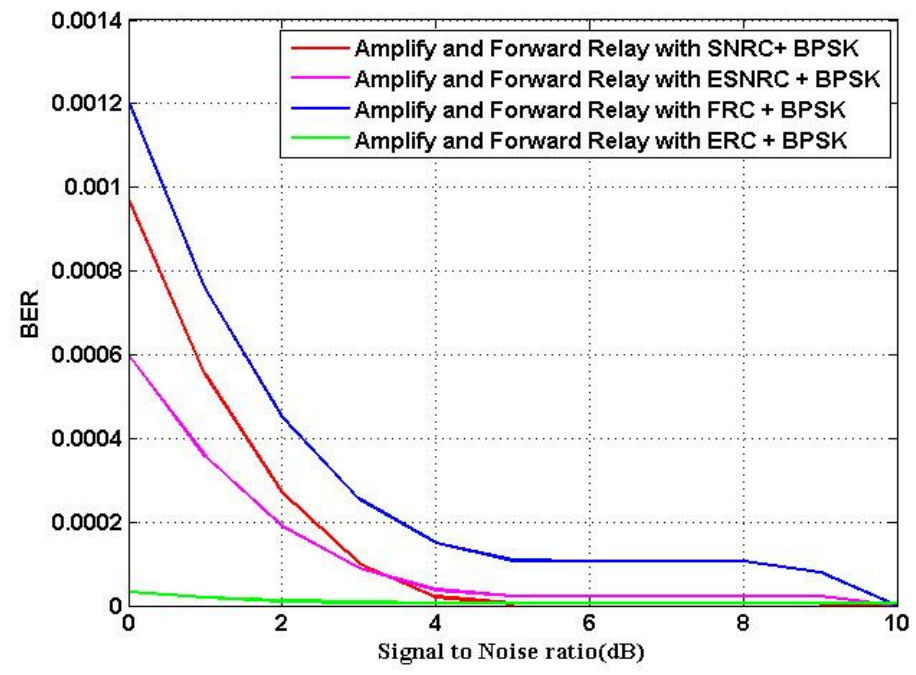

Figure 2: Performance simulations of the Cooperative OFDM wireless communication system with implementation of Amplify and Forward relaying protocol under

four signal combining schemes and BPSK digital modulation 


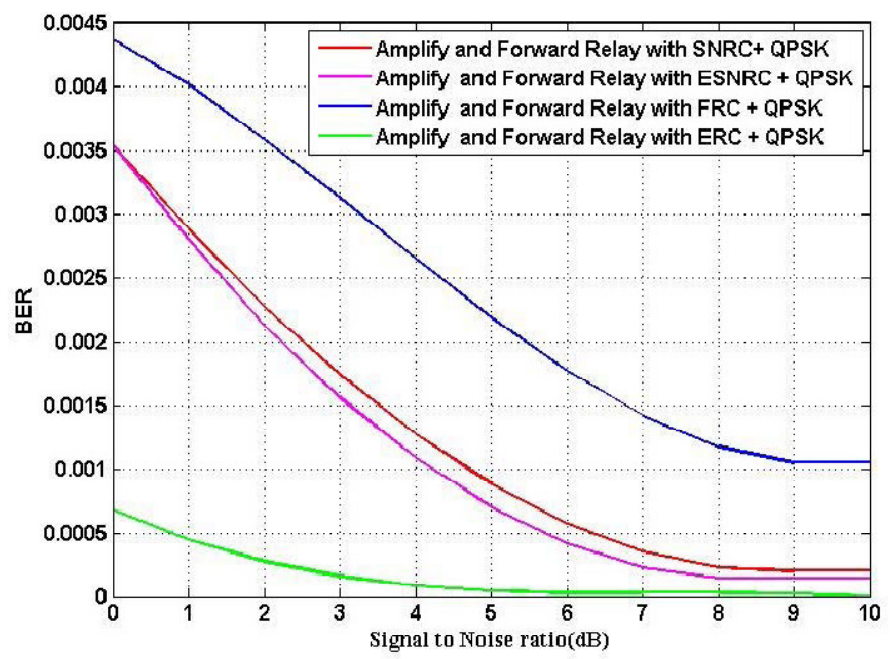

Figure 3: Performance simulations of the Cooperative OFDM wireless communication system with implementation of Amplify and Forward relaying protocol under four signal combining schemes and QPSK digital modulation

On critical evaluation on simulation results presented in Figure 5, the system shows a satisfactory performance under Equal Ratio Combining scheme with DAF relaying protocol and QPSK digital modulation. For a typically assumed SNR value of $4 \mathrm{~dB}$, the BER value is 0.0001 which is high as compared to 0.00001 in case of BPSK digital modulation(Figure 4).Figure 6 through Figure 8 show the transmitted and retrieved color image at different SNR values for the Cooperative OFDM system under implementation of Amplify and Forward relaying protocol with Equal Ratio Combining and BPSK digital modulation schemes.

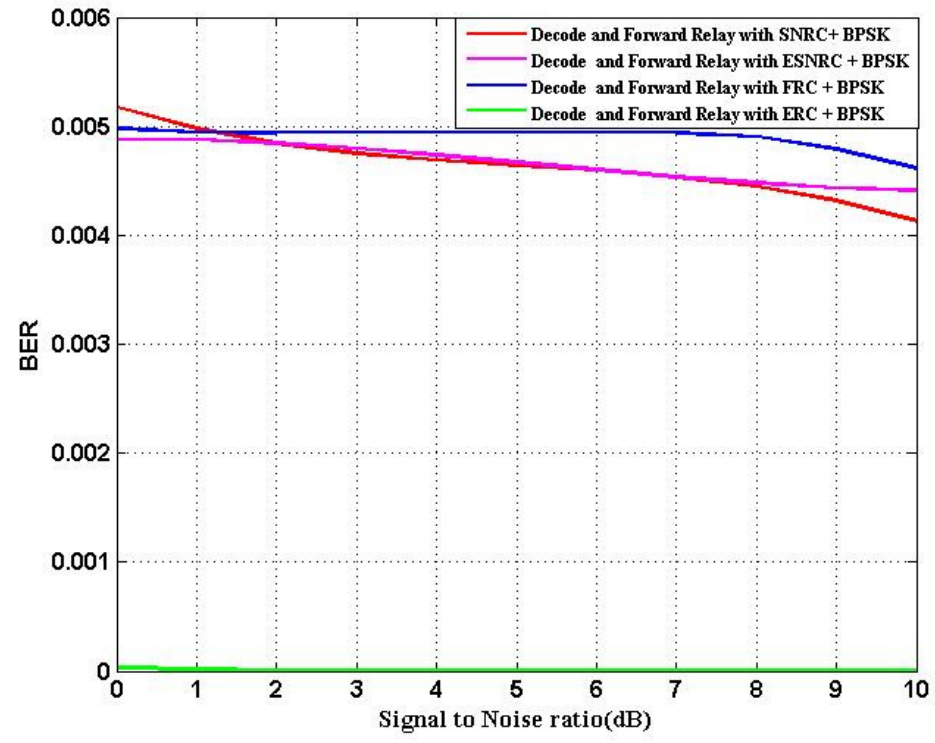

Figure 4: Performance simulations of the Cooperative OFDM wireless communication system with implementation of Decode and Forward relaying protocol under

four signal combining schemes and BPSK digital modulation 
International Journal of Information Technology Convergence and Services (IJITCS) Vol.1, No.3, June 2011

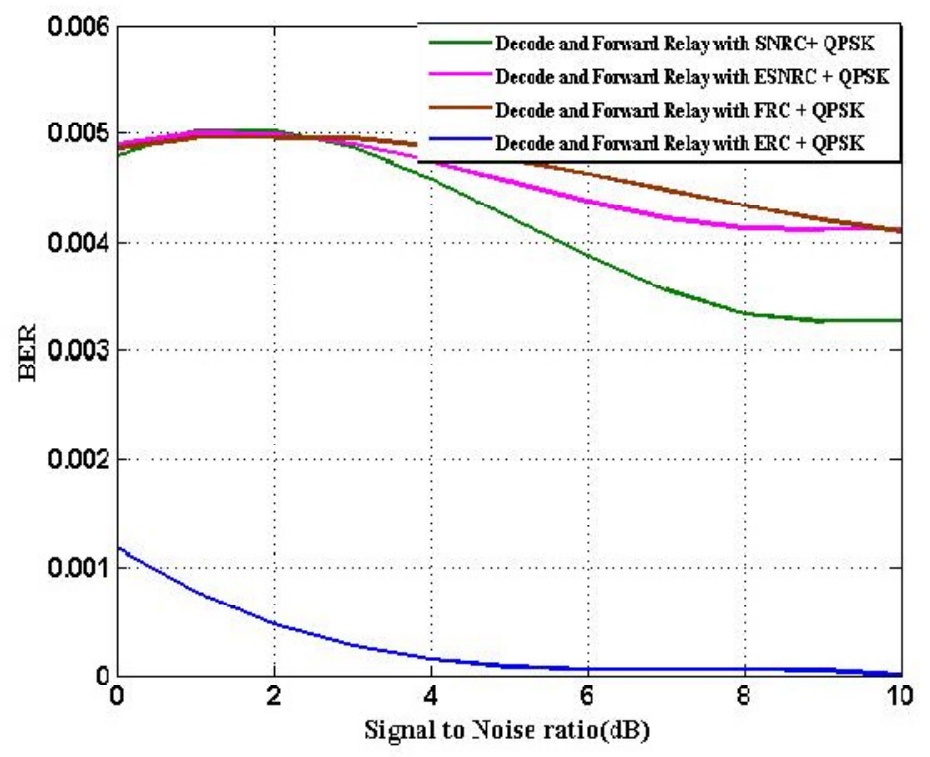

Figure 5: Performance simulations of the Cooperative OFDM wireless communication system with implementation of Decode and Forward relaying protocol under

four signal combining schemes and QPSK digital modulation

Transmitted image

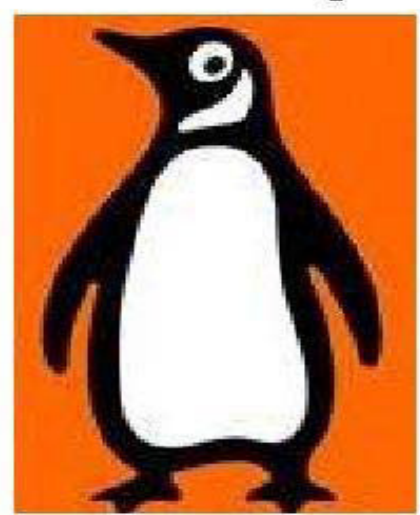

Retrieved image

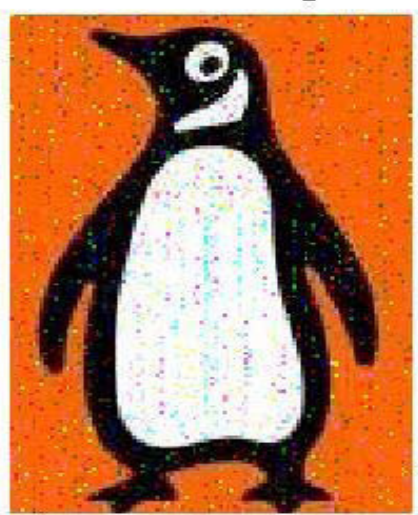

Figure 6: Transmitted and Retrieved color image at $0 \mathrm{~dB}$ SNR for the Cooperative OFDM wireless communication system under implementation of Amplify and Forward relaying protocol with Equal Ratio Combining and BPSK

Digital modulation schemes 

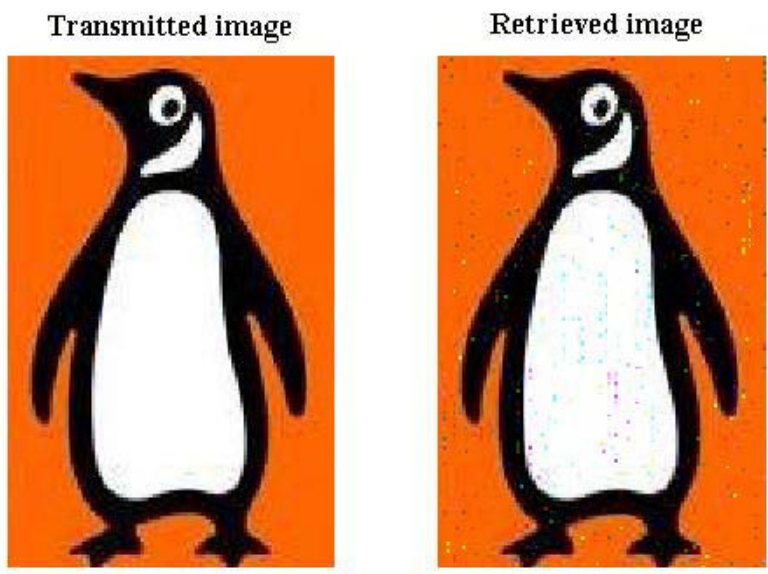

Figure 7: Transmitted and Retrieved color image at $4 \mathrm{~dB}$ SNR for the Cooperative OFDM wireless communication system under implementation of Amplify and Forward relaying protocol with Equal Ratio Combining and BPSK

digital modulation schemes

Transmitted image

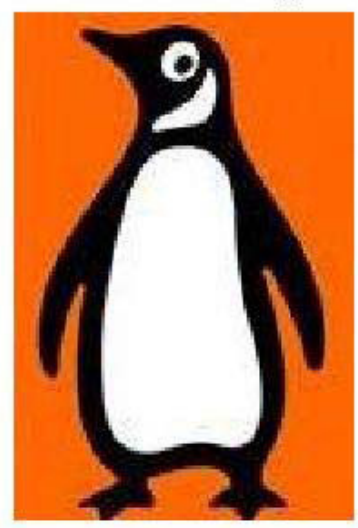

Retrieved image

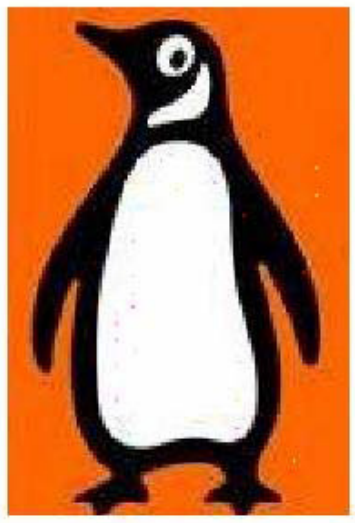

Figure 8: Transmitted and Retrieved color image at $10 \mathrm{~dB}$ SNR for the Cooperative OFDM wireless communication system under implementation of Amplify and Forward relaying protocol with Equal Ratio Combining and BPSK digital modulation schemes 
International Journal of Information Technology Convergence and Services (IJITCS) Vol.1, No.3, June 2011

\section{Conclusion}

In this paper, we have presented simulation results concerning the adaptation of various signals combining and relaying protocol schemes in a Cooperative OFDM wireless communication system. A range of system performance results highlights the impact of signal combining scheme and relaying protocol on color image transmission.. In the context of system performance, it can be concluded that the implementation of BPSK digital modulation technique in Amplify and Forward relaying protocol with Equal Ratio signal combining scheme provides satisfactory result for such a cooperative OFDM communication system.

\section{References}

[1] Peter Hong, Y.W, Huang, Wan-Jen and Jay Kuo, C.C, 2010:Cooperative Communications and Networking, Technologies and System Design, Springer Science and Business Media, LLC, New York, DOI 10.1007/978-1-4419-7194-4.

[2] Young-Han Nam, Lingjia Liu, Yan Wang, Charlie Zhang, Joonyoung Cho and Jin-Kyu Han, 2010: Cooperative Communication Technologies for LTE-Advanced, IEEE International Conference on Acoustic Speech and Signal Processing (ICASSP), 5610-5613.

INSPEC Accession Number: 11541040, DOI:10.1109/ICASSP.2010.5495250

[3] Sendonaris, A, Erkip, E, and Aazhang,B, 2003(a):User Cooperation diversity -Part I: System Description, IEEE Transactions on Communications , 51(11): 1927-1938.

INSPEC Accession Number: 7800539, : DOI:10.1109/TCOMM.2003.818096

[4] Sendonaris, A, Erkip, E, and Aazhang, B, 2003(b): User Cooperation diversity - Part II:

Implementation aspects and performance analysis, IEEE Transactions on Communications, 51(11): 1939-1948.

INSPEC Accession Number: 7800540, DOI: 10.1109/TCOMM.2003.819238

[5] Laneman, J. N., Tse ,D. N. C., and Wornell ,G. W., 2004:Cooperative diversity in wireless networks: efficient protocols and outage behavior, IEEE Transactions on Information . Theory, 50(12): 3062-3080. INSPEC Accession Number: 8208822, DOI: 10.1109/TIT.2004.838089

[6]Leng Zhi-juan, Cheng En, Sun Hai-xin, Zhu Qing-kun, Chen Vi-long, 2010: The Research Of The Cooperative OFDM System, 2nd International IEEE Conference on Industrial Mechatronics and Automation (ICIMA),2:582-585, ISBN: 978-1-4244-7653-4,DOI: 10.1109/ICINDMA.2010.5538239

[7] Tomáš, K., 2003: Utilization of MATLAB for Digital Image Transmission Simulation Using the DVB Error Correction Codes, Radio Engineering, 12: 31-36.

[8]. Goldsmith, Andrea , 2005:Wireless Communications, First Edition, Cambridge University Press, United Kingdom 
Shaikh Enayet Ullah is a Professor of the Department of Applied Physics and Electronic Engineering, Faculty of Engineering, University of Rajshahi, Bangladesh. He received his B.Sc(Hons) and M.Sc degrees in Applied Physics and Electronics in 1983 and 1985 respectively from University of Rajshahi. He received his Ph.D degree in Physics from Jahangirnagar University, Bangladesh in 2000. He is now working as a Professor and Chairman (on deputation) in the Department of Information and Communication Engineering, University of Rajshahi. He has published more than 45 papers in multidisciplinary fields. His main research interests include Cooperative communications, MIMO-OFDM, WiMAX and LTE-Advanced.

Mafruha Mowrin Hossain was born in Rajshahi, Bangladesh. She received her B.Sc (Hons ) and M.Sc degrees in Information and Communication Engineering(ICE) in 2009 and 2011 from University of Rajshahi , Bangladesh. She is engaged with Research work with Professor Dr. Shaikh Enayet Ullah. Her main reaseach interests include Cooperative Commun-ications and MIMO-OFDM.
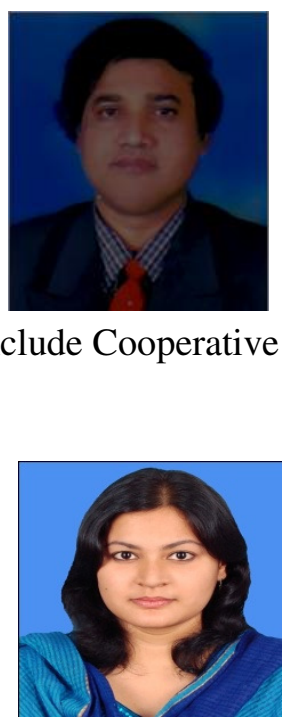